\title{
ARTICLE Glutamatergic hypo-function in the left superior and middle temporal gyri in early schizophrenia: a data-driven three-dimensional proton spectroscopic imaging study
}

Juan R. Bustillo ${ }^{1,2}$, Joel Upston ${ }^{1,3}$, Elizabeth Grace Mayer ${ }^{1,3}$, Thomas Jones ${ }^{1}$, Andrew A. Maudsley (iD ${ }^{4}$, Charles Gasparovic ${ }^{5}$, Mauricio Tohen ${ }^{1}$ and Rhoshel Lenroot ${ }^{1}$

Proton magnetic resonance spectroscopy ('H-MRS) studies have examined glutamatergic abnormalities in schizophrenia, mostly in single voxels. Though the critical brain nodes remain unknown, schizophrenia involves networks with broad abnormalities. Hence, glutamine plus glutamate (Glx) and other metabolites were examined with whole-brain ${ }^{1} \mathrm{H}-\mathrm{MRS}$, in early schizophrenia. Three dimensional ${ }^{1} \mathrm{H}$-MRS was acquired in young schizophrenia subjects $(N=36,19$ antipsychotic-naïve and 17 antipsychotic-treated) and healthy controls ( $\mathrm{HC}, N=29$ ). Glx (as well as $N$-acetylaspartate, choline, myo-inositol and creatine) group contrasts from all individual voxels that met spectral quality, were analyzed in common brain space, followed by cluster-corrected level alpha-value $(C C L A V \leq 0.05)$. Schizophrenia subjects had lower Glx in the left superior (STG) and middle temporal gyri $(16$ voxels, CCLAV $=0.04)$ and increased creatine in two clusters involving left temporal, parietal and occipital regions (32, and 18 voxels, CCLAV $=0.02$ and 0.04 , respectively). Antipsychotic-treated and naïve patients (vs HC) had similar Glx reductions (8/16 vs $10 / 16$ voxels respectively, but CCLAV's > 0.05). However, creatine was higher in antipsychotic-treated vs HC's in a larger left hemisphere cluster (100 voxels, $C C L A V=0.01$ ). Also in treated patients, choline was increased in left middle frontal gyrus (18 voxels, $C C L A V=0.04)$. Finally in antipsychotic-naive patients, NAA was reduced in right frontal gyri ( 19 voxels, CCLAV $=0.05$ ) and myo-inositol was reduced in the left cerebellum (34 voxels, CCLAV $=0.02$ ). We conclude that data-driven spectroscopic brain examination supports that reductions in Glx in the left STG may be critical to the pathophysiology of schizophrenia. Postmortem and neuromodulation schizophrenia studies focusing on left STG, may provide critical mechanistic and therapeutic advancements, respectively.

Neuropsychopharmacology (2020) 45:1851-1859; https://doi.org/10.1038/s41386-020-0707-y

\section{INTRODUCTION}

The N-methyl-D-aspartate receptor (NMDAR) hypo-function model of schizophrenia originated from pharmacological studies documenting emergence of psychotic-like symptoms in healthy volunteers exposed to the NMDA-blocker ketamine [1]. Though NMDA-blockers reduce the post-synaptic effect of glutamate in single neurons, in the rat, systemic ketamine increases extracellular glutamate in frontal cortex [1]. This paradoxical increment in glutamate release with ketamine, has been postulated to result from higher sensitivity of NMDA receptors in GABAergic interneurons than in pyramidal neurons, resulting in disinhibition of pyramidal neurons and increase in presynaptic glutamate release in cortical fields [1]. However, in a chronic condition like schizophrenia, persistently increased glutamate release could result in dendritic and axonal damage, with subsequent reductions in glutamate concentrations as well as in tissue volumes. Consistent with this view, longitudinal MRI studies early in the illness, have documented brain tissue reductions [2].

The ${ }^{1} \mathrm{H}$-MRS schizophrenia glutamate literature remains unclear. Three meta-analyses have examined measurements of glutamine and glutamate, all with the single-voxel approach. One reported increased medial frontal glutamine and reduced glutamate regardless of stage of illness [3]. Another reported increased glutamate, glutamine and glutamate + glutamine (Glx), in the "basal ganglia" regardless of age or medication [4]. Finally, in never-medicated schizophrenia, no abnormalities were found [5]. However, in schizophrenia, a disease with subtle but broad gray and white matter involvement and progressive structural changes [2], proton MR spectroscopic imaging ( $\left.{ }^{1} \mathrm{H}-\mathrm{MRSI}\right)$ enables measurement in much larger brain regions, thereby reducing the bias of voxel selection intrinsic to single-voxel studies.

Here we report the novel use of 3 -dimensional ${ }^{1} \mathrm{H}$-MRSI with a short echo-time (TE) to measure Glx and other metabolites of interest early in schizophrenia. Consistent with the NMDAR hypofunction model and supported by reports of elevated GlX in $\mathrm{dAC}$ by us [6] and by others in the dorsal striatum [7], we hypothesized increased Glx in schizophrenia in these structures.

\section{MATERIALS AND METHODS}

Subjects

Schizophrenia patients (SP) were recruited from the University of New Mexico (UNM) Hospitals. Inclusion criteria were: (1) DSM-5

\footnotetext{
${ }^{1}$ Departments of Psychiatry, University of New Mexico, Albuquerque, NM 87131-0001, USA; ${ }^{2}$ Department of Neurosciences, University of New Mexico, Albuquerque, NM, USA; ${ }^{3}$ Department Mathematics \& Statistics, University of New Mexico, Albuquerque, NM, USA; ${ }^{4}$ Department of Radiology, University of Miami, FL, NM, USA and ${ }^{5}$ Mind Research Network, Albuquerque, NM, USA

Correspondence: Juan R. Bustillo (jbustillo@salud.unm.edu)
}

Received: 6 March 2020 Revised: 23 April 2020 Accepted: 6 May 2020

Published online: 13 May 2020 
schizophrenia/schizophreniform disorder (SCID-DSM-5); 2) Age 1640. Exclusion criteria were diagnosis of neurological disorder or current substance use disorder (except for nicotine). Healthy controls $(\mathrm{HC})$ recruited from the community were excluded if they had: (1) any current DSM-5 disorder (except for nicotine use); (2) first-degree relatives with any psychotic disorder; (3) history of neurological disorder. The study was approved by the UNM Institutional Review Board. Subjects gave written informed consent and were reimbursed for their participation.

Neuropsychological and clinical assessments

SPs and $\mathrm{HCs}$ completed the Measurement-and-TreatmentResearch-to-Improve-Cognition-in-Schizophrenia (MATRICS) [8] battery. Patients were assessed with the Positive-and NegativeSyndrome-Scale (PANSS [9]).

\section{Magnetic resonance studies}

Acquisition. Subjects underwent an MR study at 3T using a TIM Trio scanner with a 32 channel head-coil. High resolution T1weighted imaging was carried out using a 5-echo multi-echo MPRAGE $[T E=1.64,3.5,5.36,7.22,9.08 \mathrm{~ms}$, TR (repetition time) $=$ $2530 \mathrm{~ms}$, TI (inversion time) $=1200 \mathrm{~ms}, 7^{\circ}$ flip angle, number of excitations $=1$, slice thickness $=1 \mathrm{~mm}$, FOV (field of view) $=256$ $\mathrm{mm}$, resolution $=256 \times 256]$.

Whole-brain ${ }^{1} \mathrm{H}-\mathrm{MRSI}$ was acquired using the EPSI sequence which uses echo-planar acquisition after spin-echo excitation $\left[\mathrm{TE}=17.6 \mathrm{~ms}, \mathrm{TR}=1551 \mathrm{~ms}, \operatorname{TR}\left(\mathrm{H}_{2} \mathrm{O}\right)=511 \mathrm{~ms}\right.$, non-selective lipid inversion nulling with $\mathrm{TI}=198 \mathrm{~ms}$, FOV $=280 \times 280 \times 180 \mathrm{~mm}$, $50 \times 50 \times 18 \mathrm{k}$-space samples, echo-train length $=1000$ points, bandwidth $=2500 \mathrm{~Hz}$, reduced k-space sampling (acceleration factor $=0.7$ ), and acquisition time $=17 \mathrm{~min}$ ] [10]. EPSI included a water reference measurement that was interleaved with the metabolite signal acquisition.

\section{General analytic approach}

Although we collected whole-brain EPSI data, not all data met spectral quality criteria. Hence, a systematic, approach was followed to compromise between the maximum amount of data per subject, maintaining spectral quality and minimizing falsepositive rates (FPR). Because ${ }^{1} \mathrm{H}$-MRS metabolites vary greatly by brain tissue composition [11], for statistical analyses we selected AFNI since it allowed us covariation of gray/gray-plus-white matter at the voxel level [12]. However, to use this feature all subjects must provide data for each voxel analyzed. Hence, with this approach, imputation of some missing data was necessary, as in other neuroimaging modalities [13]. Furthermore, inclusion of some suboptimal spectral data in voxels mainly surrounded by best spectra, was also implemented (see Supplementary Table 1 for spectral quality metrics). This is in part justified by the relatively high smoothing of these data such that each spectral value is affected by the surrounding voxels' values. We mitigated the FPR in the statistical levels 1 and 2 analyses described below. The processing pipeline is summarized in Fig. 1.

\section{Reconstruction}

EPSI reconstruction was carried out using the MIDAS package as previously described [14]. Processing included corrections for $B_{0}$ shifts, generation of white-matter, gray-matter, and CSF tissue segmentation maps using FSL FAST, lipid k-space extrapolation and linear registration of the T1-weighted MR images to the EPSI images. Data was acquired using a spatial oversampling approach [15] that obtains data with a relatively small nominal voxel volume of $0.31 \mathrm{~cm}^{3}$ which following application of $B_{O}$ correction and convolution smoothing resulted in an effective voxel volume of $1.55 \mathrm{~cm}^{3}$. Following mild spectral smoothing of $2 \mathrm{~Hz}$ (which has minimal effect on quantification accuracy [16]), spectra were analyzed using a time-frequency parametric modeling method [17] that incorporated a-priori information on the resonance peaks and a Gaussian line-shape model. Metabolite maps were interpolated to $64 \times 64 \times 32$ points (voxel size $4.375 \times 4.375 \times$ $5.625 \mathrm{~mm}$ ). Correction for frequency shifts due to $B_{0}$ inhomogeneity was done using two steps. First, the spectra were frequency and phase corrected using a standard eddy-current correction procedure with the time-domain phase correction function obtained from the water reference EPSI. Second, following spectral analysis the final data were corrected for the phase and frequency offset values obtained from the fitting.

\section{Registration}

EPSI water reference measurement was spatially registered to the MPRAGE T1-weighted image with MIDAS-MSREG [14].

Spectral fitting

The fitted metabolite values from the corrected spectra were estimated using MIDAS-FITT2 program [14] for $\mathrm{N}$-acetyl-aspartate (NAA), total creatine (creatine and phosphocreatine, denoted t-Cr), total choline (choline, glycerol-phospho-choline, and phos-phocholine, denoted t-Cho), myo-inositol, lactate and glutamate plus glutamine (denoted Glx; see Fig. 1 for example of fitted spectrum). However, due to sensitivity to noise at the voxel level lactate was not analyzed.

The amplitude of macromolecular resonances were considered negligible due to use of lipid inversion nulling during the acquisition. Still, any residual signal was part of the spectral baseline that was characterized using a wavelet smoothing approach incorporated into the spectral model [10]. Individual metabolite maps were signal normalized and corrected for any bias-field variations using the signal from the water reference measurement. This water signal was corrected for tissue water density based on the fractional tissue volume obtained from the down-sampled tissue segmentation maps and converted to $100 \%$ water equivalent based on the assumption of tissue water content in gray-matter, white-matter, and CSF of $79 \%, 70 \%$, and $98 \%$, respectively [11]. Results were not corrected for relaxation rates and represent institutional units.

\section{Warping}

The individual metabolite maps were exported from MIDAS and warped using SPM12's Normalize function into MNI space. In this process the output voxel size is set to the spectroscopic voxel size $(4.375 \times 4.375 \times 5.625 \mathrm{~mm})$. We did not set to a usual smaller grid (eg.: $1 \times 1 \times 1 \mathrm{~mm}$ ) in order to minimize interpolation. Two warping steps were done as per https://www.fil.ion.ucl.ac.uk/ spm/doc/spm8_manual.pdf. First, using the individual subject gray matter map in spectroscopic imaging space, a linear 12-parameter affine transformation was done to account for differences in position. Then a second non-linear warping was done to account for small anatomical differences. These warped maps were then visually inspected to insure a quality alignment using SPM12's Check Reg function.

\section{Quality filtering}

To correct for partial volume effects, the metabolite maps are divided by $1-f_{\mathrm{CSF}}$, where $f_{\mathrm{CSF}}$ is the fraction of CSF in each voxel. To ensure quality of the metabolite values from spectra, the metabolite maps were filtered to include spectra that met three criteria: overall linewidths between 2 and $12 \mathrm{~Hz}$ (inclusive); Cramér-Rao lower bound (CRLB) for spectral fitting of each metabolite of $\leq 20 \%$; and $f_{\mathrm{CSF}} \leq 0.3$ [18] (these were labeled best spectra). Spectra that failed to meet these qualifications were split into two groups, poor and intermediate spectra. Intermediate spectra had: linewidths greater than 1 and less than or equal to 16; CRLB between 1 and 99, inclusively; $f_{\mathrm{CSF}} \leq 0.3$; and at least 18 of the 26 nearest neighboring voxels (directly touching the voxel) were best spectra. Not all 26 surrounding voxels were required to be best spectra, because when intermediate spectra bordered CSF 
Glutamatergic hypo-function in the left superior and middle temporal gyri... JR Bustillo et al.
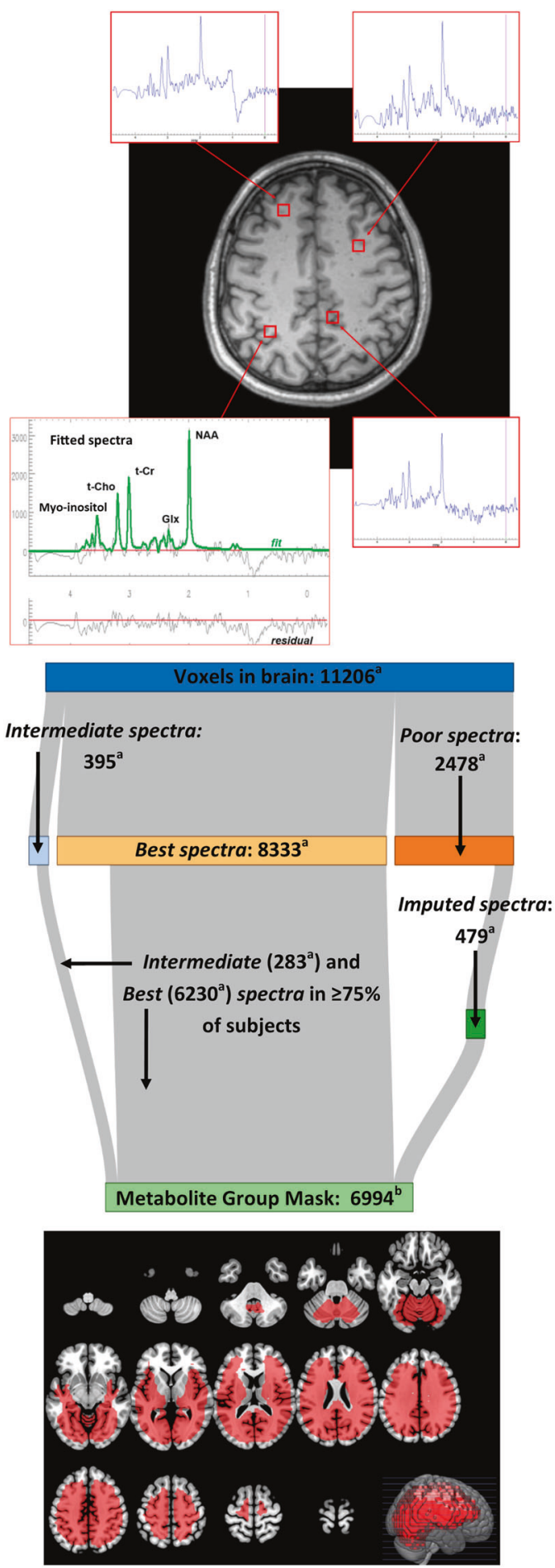

GLX Metabolite Group Mask

${ }^{\text {a }}$ Average number of voxels in spectroscopic space, per subject for Glx in the schizophrenia group.

${ }^{\mathrm{b}}$ Number of voxels in spectroscopic space in the Glx Metabolite Group Mask.

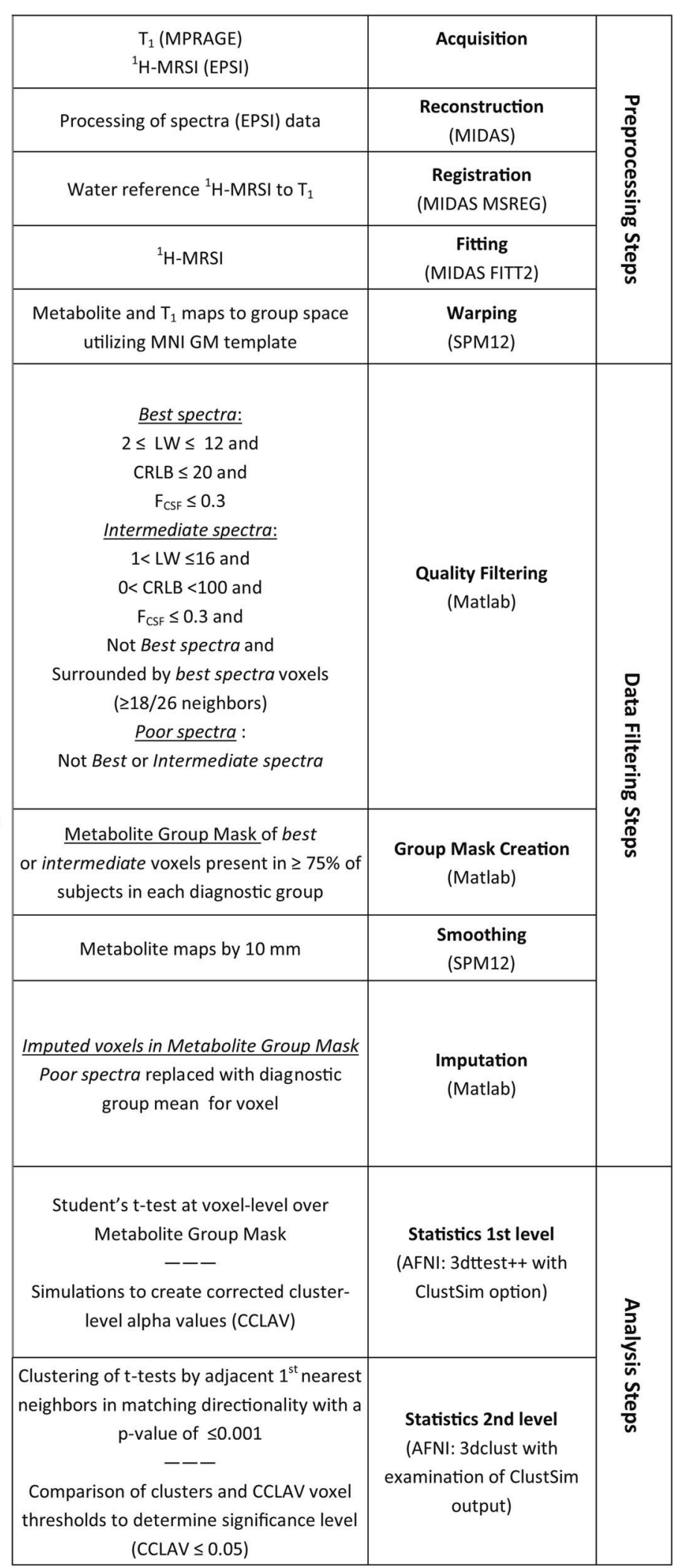

Fig. 1 Summary of data processing steps (two rightmost columns, include software used), with corresponding descriptions (to the left) and representative illustrations. NAA is $\mathrm{N}$-acetyl-aspartate, Glx is glutamate plus glutamine, $\mathrm{t}$ - $\mathrm{Cr}$ is creatine plus phosphocreatine and t-Cho is choline, glycerol-phospho-choline, plus phos-phocholine. 
1854

spaces, about one third of neighboring voxels were expected to have poor spectra due to $f_{\mathrm{CSF}}>0.3$. Intermediate spectra were considered to have some potential useful spectral information and hence their metabolite values were kept. All others were considered poor spectra and their metabolite values were removed.

\section{Metabolite group mask}

This mask was composed only of the best and intermediate spectra voxels that were present in at least $75 \%$ of subjects in each diagnostic group. We chose this $75 \%$ threshold through analyzing the distribution of voxel counts relative to possible thresholds for each metabolite in the data. As seen in Supplementary Fig. 2, both Glx and myo-inositol were the metabolites with the lowest median (75\%) for the percentage of subjects with best or intermediate spectra in the metabolite group mask. This is expected since these are the two metabolites harder to fit. Hence, the $75 \%$ threshold was selected for the five group masks. Missing subject values in the metabolite group masks (Supplementary Fig. 3) were later imputed as described below.

\section{Smoothing}

These metabolite group masks were then smoothed using a spatially stationary Gaussian filter with a kernel width of $10 \mathrm{~mm}$ in SPM12 to minimize potential spatial warping errors [11]. The effective smoothing of the spectroscopic data in the $X-Y$ plane is $15.8 \mathrm{~mm}$ and in the $Z$-plane is $19.6 \mathrm{~mm}$.

\section{Imputation}

Because up to $25 \%$ of subjects in each diagnostic group did not have a particular voxel value in the metabolite group mask, the metabolite values for the poor spectra voxels were imputed using the diagnostic group (SP, $\mathrm{HC}$ ) average concentration for that voxel. As the percentage of imputed voxels were clearly $<10 \%$ (see Supplementary Table 1, row: Imputed voxels/Metabolite_Group_Mask) on average across all metabolites and groups, the mean substitution imputation approach we selected, outperforms the removal of missing data [19]. This was done to insure we were reasonably preserving the maximum number of voxels possible for the analysis, without adding variability to the populations' metabolite concentrations. Imputations only accounted for an average of between $2 \%$ and $7 \%$ of the total spectra in each metabolite group mask. We did not use AFNI's 3dMEMA function, which can handle missing voxels, because it does not allow voxel level covariates (see:"https://afni.nimh.nih.gov/MEMA").

\section{Statistical analyses, Level 1}

The main analyses examined group differences separately for each of the five metabolites of interest with adjustments made on the voxel level for gray matter proportion $\left(\frac{G M}{G M+W M}\right)$ and for age at the subject level, factors known to have large effects on metabolite's variability [11]. AFNl's 3dttest++ and 3dclustpackages were used which support subject and voxel-level covariates [12]. 3dttest++ implemented Student t-tests with an assumed equal variance for all voxels that fell within each metabolite group mask. Due to the significant smoothing of the metabolite data, we minimize FPR with permutation testing of 3dttest++'s ClustSim option [20]. ClustSim computed cluster-size thresholds using 10000 simulated noise-only t-tests for a more accurate spatial autocorrelation function of the noise. ClustSim then estimated the probability of false positive clustering given the sample and corrected the voxel clustering thresholds to account for this.

Statistical analyses, Level 2

The resulting maps were clustered using $3 d c l u s t$ following three criteria: (1) each voxel differed between the groups with $p$-value $\leq$ 0.001 ; (2) group differences for each voxel in the cluster were in the same direction; and (3) voxels were nearest neighbors (faces touching). The corrected clustering thresholds from ClustSim were compared to the number of voxels clustered in 3dclust to insure the cluster met a corrected cluster-level alpha-value (CCLAV) of $\leq 0.05$.

Finally, Spearman's Rho tested correlations between concentrations that differed between groups and clinical and cognitive assessments.

\section{RESULTS}

Demographics

Thirty-six SPs and $29 \mathrm{HCs}$ participated (Table 1). Subject were similarly young (age/years, $\mathrm{SP}=22.7 \pm 3.9, \mathrm{HC}=23 \pm 4.4, p=$ 0.78 ), with no significant differences in gender, race, cardiovascular risk, tobacco use and history of substance use disorders, except for cannabis. SP had greater diagnosis of past cannabis use $(p=0.01)$. SP also had worse personal $(p<0.001)$ and parental socioeconomic status (SES; $p=0.002$ ) and lower MATRICS overall $t$-score $(p=0.001)$. Finally, $19 \mathrm{SP}$ were naïve to antipsychotic medications and 17 were treated. These subgroups did not differ in age of onset of psychosis, substance use histories, tobacco use, positive and negative symptoms severity, MATRICS overall t-score, age or the other demographic variables. The naïve subgroup had a lower number of psychiatric hospitalizations than the treated SP $(0.28 \pm 0.5$ vs $1.3 \pm 1.3$, respectively, $p=0.002)$.

Table 1. Demographic and clinical characteristics.

\begin{tabular}{|c|c|c|c|}
\hline & $\begin{array}{l}\text { Healthy } \\
\text { controls } \\
(n=29) \\
\text { Mean } \pm \text { SD }\end{array}$ & $\begin{array}{l}\text { Schizophrenia } \\
(n=36) \\
\text { Mean } \pm \text { SD }\end{array}$ & $p$-value \\
\hline Age (years) & $23 \pm 4.4$ & $22.8 \pm 3.9$ & 0.78 \\
\hline Gender (male/female) & $17 / 12$ & $24 / 12$ & 0.5 \\
\hline $\begin{array}{l}\text { Race (Caucasian/ } \\
\text { black/native) }\end{array}$ & $26 / 1 / 2$ & $32 / 3 / 1$ & 0.5 \\
\hline $\mathrm{SES}^{\mathrm{a}}$ & $4.1 \pm 1.2$ & $5.9 \pm 1.5$ & 0.001 \\
\hline Familial SES & $2.9 \pm 1.6$ & $4.4 \pm 1.8$ & 0.002 \\
\hline Vascular risk score ${ }^{\mathrm{b}}$ & $4.0 \pm 0.2$ & $4.1 \pm 0.2$ & 0.7 \\
\hline $\begin{array}{l}\text { MATRICS-overall } \\
\text { T score }\end{array}$ & $48.4 \pm 5.9$ & $34.0 \pm 9.2$ & 0.001 \\
\hline Smoker (yes/no) & $4 / 25$ & $8 / 28$ & 0.55 \\
\hline Alcohol (yes/no) & $1 / 28$ & $3 / 33$ & 0.8 \\
\hline Cannabis (yes/no) & $1 / 28$ & $18 / 18$ & 0.01 \\
\hline Stimulant (yes/no) & $0 / 29$ & $2 / 34$ & 0.34 \\
\hline Opioid (yes/no) & $0 / 29$ & $1 / 35$ & 0.5 \\
\hline Sedative (yes/no) & $0 / 29$ & $0 / 36$ & 1.0 \\
\hline Cocaine (yes/no) & $0 / 29$ & $2 / 34$ & 1.0 \\
\hline $\begin{array}{l}\text { Hallucinogens (yes/ } \\
\text { no) }\end{array}$ & $0 / 29$ & $2 / 34$ & 0.63 \\
\hline $\begin{array}{l}\text { Psychosis onset } \\
\text { (years) }\end{array}$ & & $20.5 \pm 4.8$ & - \\
\hline Positive symptoms & & $16.9 \pm 5.0$ & - \\
\hline Negative symptoms & & $15.7 \pm 5.5$ & - \\
\hline Antipsychotic (yes/no) & & $17 / 19$ & - \\
\hline $\begin{array}{l}\text { Antipsychotic dose } \\
(\mathrm{mg})^{\mathrm{c}}\end{array}$ & & $4.9 \pm 7.1$ & - \\
\hline
\end{tabular}




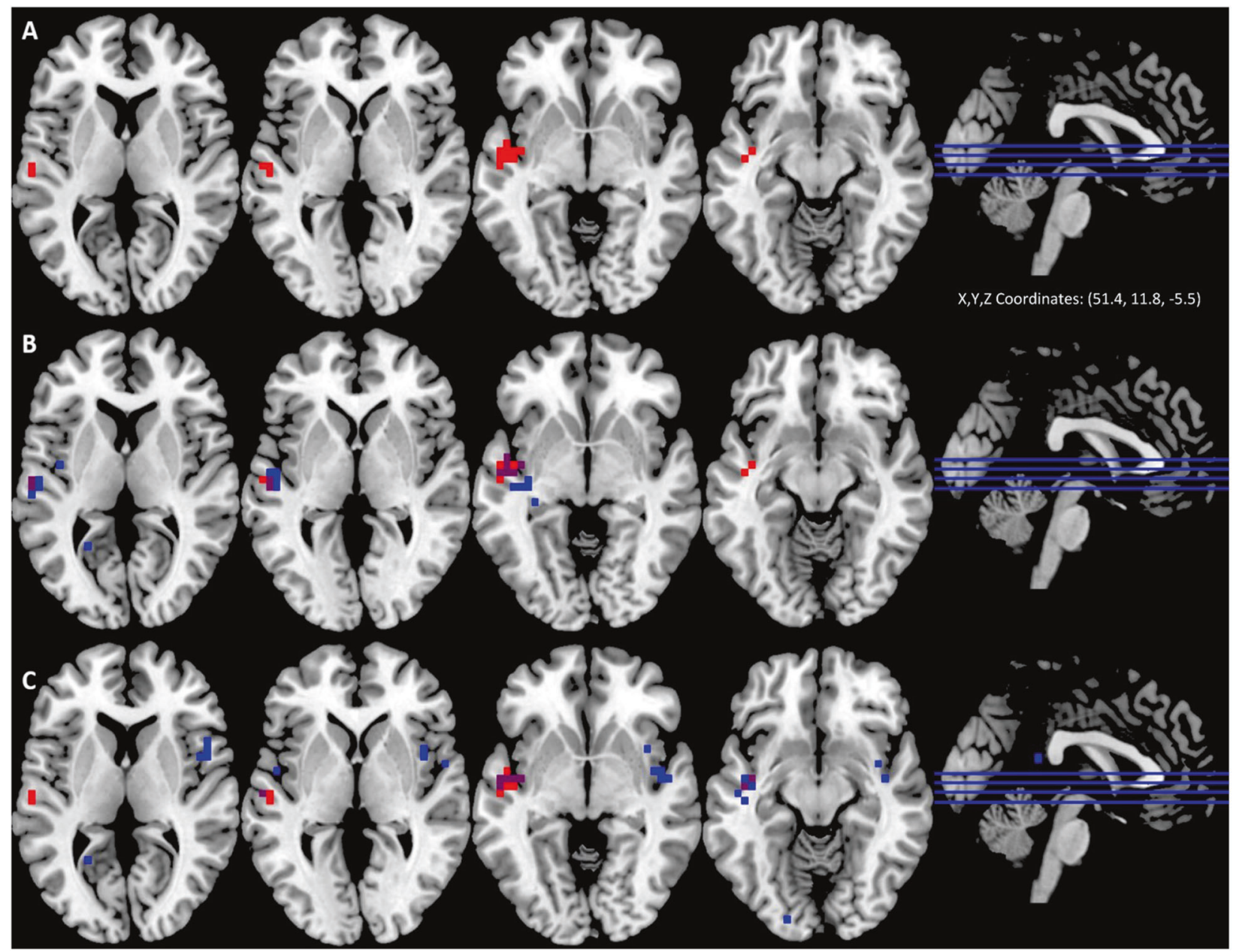

Fig. 2 Reduced glutamine plus glutamate (Glx) in schizophrenia. Row a Reduced Glx (shown in red) in schizophrenia (SP) compared to healthy controls $(\mathrm{HC})$ in one 16 voxel cluster $(C C L A V=0.04)$, mainly in the left superior temporal gyrus-STG and the middle temporal gyrus (MTG; see $x, y, z$, the MNI-based, cluster-center coordinates). Row b 10/16 voxels (shown in purple) have reduced Glx ( $p \leq 0.001$, at the voxellevel) in antipsychotic-naïve schizophrenia vs, healthy controls. Blue voxels also have reduced Glx but are not part of the original significant cluster, which is shown in red and purple. Row c 8/16 voxels (shown in purple) have reduced Glx ( $p \leq 0.001)$ in antipsychotic-treated schizophrenia vs, healthy controls. Blue voxels also have reduced Glx but are not part of the original significant cluster, which is shown in red and purple.

Neurometabolites differences

Glutamate+glutamine. One cluster (16 voxels) had lower Glx in $\mathrm{SP}$ vs HC's (CCLAV $=0.04$; mainly in left superior temporal gyrusSTG, middle temporal gyrus (MTG) and temporal white matter; Fig. 2a, Supplementary Fig. 1 and Supplementary Table 2). Both the antipsychotic-naïve (10/16 voxels; Fig. $2 b)$ and the antipsychotic-treated SP's (8/16 voxels; Fig. 2c) had a similar area of reduced Glx when compared to the $\mathrm{HC}$ group; however, these contrasts (eg: antipsychotic-naïve vs, HC) did not meet CCLAV. Following co-variation for history of cannabis use or parental SES the cluster differences between SP vs $\mathrm{HC}^{\prime}$ 's remained significant $(C C L A V=0.03$ and 0.03 , respectively).

\section{Total-Creatine}

There were two clusters, both with higher t-Cr in SP's vs HC's (Fig. 3a and Supplementary Fig. 1 and Supplementary Table 2). One involved 32 voxels mainly in the left STG, inferior parietal lobule (IPL), insula and superior longitudinal fasciculus (SLF). $(C C L A V=0.02)$. The second cluster involved 18 voxels mainly in the left middle temporal and angular gyri $(C C L A V=0.04)$. History of cannabis use or parental SES did not account for the clusters $(C C L A V=0.02$ and 0.02 , respectively for the first, and
$C C L A V=0.02$ and 0.04 respectively, for the second cluster). In the antipsychotic-treated subgroup, 32/32 voxels of the first cluster had higher $\mathrm{t}-\mathrm{Cr}$ vs the $\mathrm{HC}$ group (Fig. $3 \mathrm{~b}$ ), while in the antipsychoticnaïve SP's only $1 / 32$ voxels did (Fig. 3c). Also, for the second cluster, $14 / 18$ voxels had increased $\mathrm{t}-\mathrm{Cr}$ in the antipsychotic-treated group (Fig. 3b), but only $6 / 18$ did in the antipsychotic-naïve SP's (Fig. 3c; none of these contrasts met (CLAV). In order to further explore the impact of antipsychotic exposure on $\mathrm{t}-\mathrm{Cr}$, independent contrasts between each SP subgroup and $\mathrm{HC}^{\prime}$ 's were implemented. Antipsychotic-treated SP's had higher t-Cr than $\mathrm{HC}^{\prime}$ 's in three clusters: (1) a larger left hemisphere cluster (100 voxels, CCLAV = 0.01; Supplementary Fig. 1 and Supplementary Table 2) involving left insula, postcentral gyrus, STG, IPL and SLF; (2) left and right cuneus ( 51 voxels, CCLAV $=0.01$ ); and (3) left occipital ( 47 voxels, $\mathrm{CCLAV}=0.01)$. No clusters were identified when comparing antipsychotic-naïve vs $\mathrm{HC}^{\prime}$.

\section{Other metabolites}

For NAAC, t-Cho and myo-inositol, there were no clusters that passed CCLAV in the contrasts between the SP vs HC groups. However, because of the large effects of medication exposure on $\mathrm{t}-\mathrm{Cr}$, we explored this variable for the other metabolites. In 


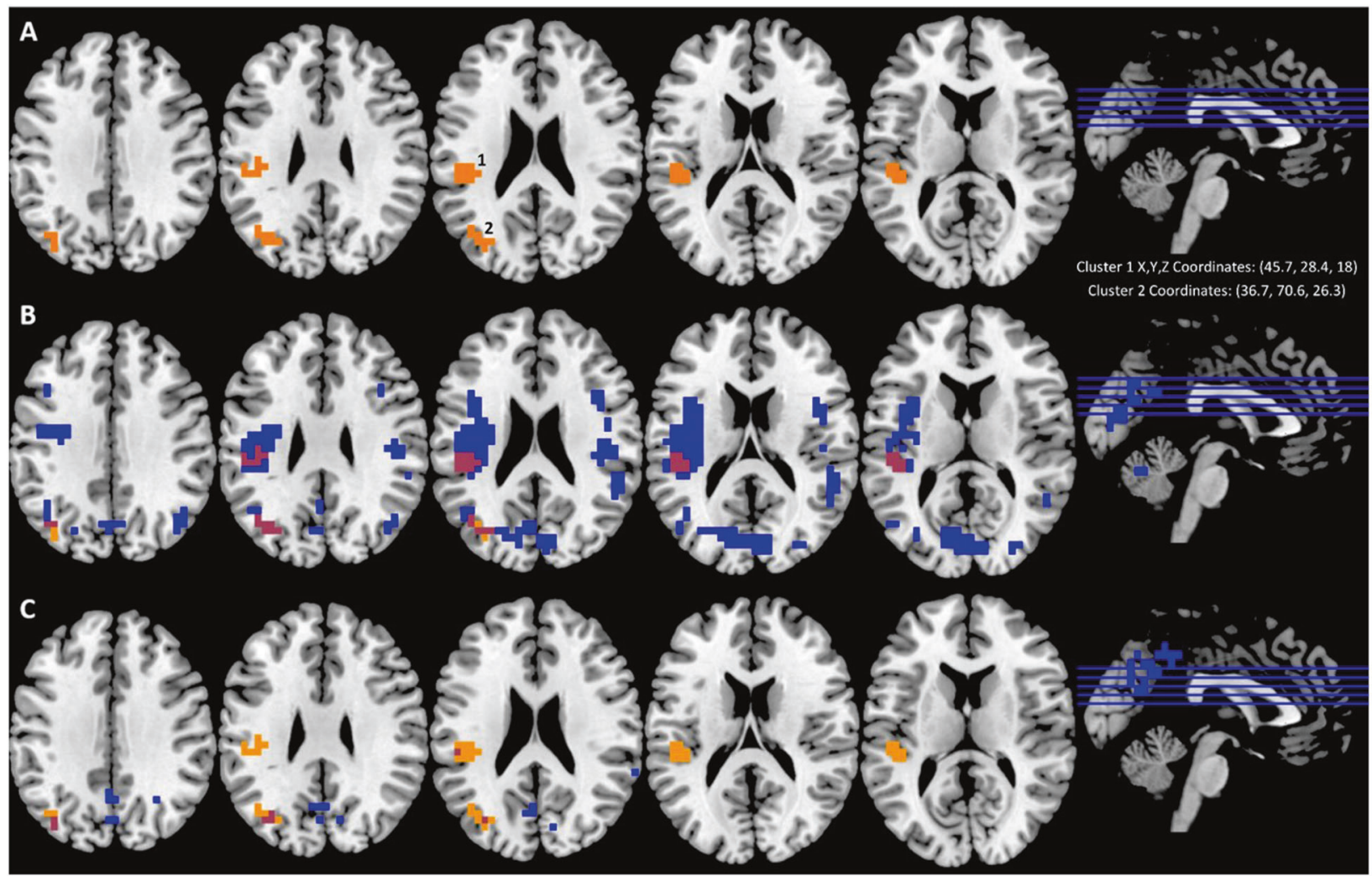

Fig. 3 Increased creatine plus phosphocreatine (t-Cr) in schizophrenia. Row a Increased t-Cr in SP compared to HC in two clusters (shown in orange): one involved 32 voxels $(C C L A V=0.02)$ mainly in the left $S T G$, inferior parietal lobule $(I P L)$ and insula. The second cluster involved 18 voxels $(C C L A V=0.04)$ mainly in the left middle temporal and angular gyri. Row b 32/32 voxels (shown in purple) in the first cluster and $14 / 18$ voxels (shown in purple) in the second cluster have reduced $\mathrm{t}-\mathrm{Cr}(p \leq 0.001$, at the voxel-level) in antipsychotic-treated schizophrenia vs, healthy controls. Blue voxels also have increased $\mathrm{t}-\mathrm{Cr}$ but are not part of the original significant clusters, which are shown in orange and purple. Row c $1 / 32$ voxels (shown in purple) in the first cluster and $6 / 18$ voxels (shown in purple) in the second cluster have reduced t-Cr ( $p \leq$ 0.001 , at the voxel-level) in antipsychotic-naive schizophrenia vs, healthy controls. Blue voxels also have increased $\mathrm{t}-\mathrm{Cr}$ but are not part of the original significant clusters, which are shown in orange and purple.

antipsychotic-treated SP's vs HC's, t-Cho was increased mainly in left middle frontal gyrus ( 18 voxels, $C C L A V=0.04$; Fig. $4 a$, Supplementary Fig. 1, and Supplementary Table 2). Finally in antipsychotic-naive patients, NAA was reduced in mainly right medial, superior frontal gyri and middle frontal white matter (19 voxels, $C C L A V=0.05$; Fig. $4 \mathrm{~b}$ ) and myo-inositol was reduced in the left cerebellum (34 voxels, $C C L A V=0.02 ;$ Fig. 4c). History of cannabis use or parental SES did not account for these cluster differences (for $\mathrm{t}-\mathrm{Cho}, \mathrm{CCLAV}=0.03$ and 0.04 , respectively; for $\mathrm{NAA}, \mathrm{CCLAV}=0.05$ and 0.04 , respectively; for myo-inositol, CCLAV $=0.02$ and 0.02 , respectively).

Finally, the neurochemicals in the clusters affected in SP's (Glx and $\mathrm{t}-\mathrm{Cr}$ ) did not correlate (Spearman's Rho) with cognition (full sample) or symptoms (only in SP).

\section{DISCUSSION}

This is the first ${ }^{1} \mathrm{H}$-MRS study to examine Glx as well as the other commonly measured neurometabolites in schizophrenia, with a data-driven 3-dimensional imaging approach that accounts for false positive rates. Contrary to our hypothesis, Glx was not increased in the dorsal anterior cingulate (due to suboptimal coverage, we could not test the dorsal striatum). Only one cluster differed between groups: the left STG/MTG had reduced Glx in SP's, and this reduction was apparent in antipsychotic-naïve as well as in medicated patients, though the subgroup comparisons were not statistically significant. The only other neurometabolite affected was $\mathrm{t}-\mathrm{Cr}$, which was higher in $\mathrm{SP}^{\prime} \mathrm{s}$ than $\mathrm{HC}^{\prime} \mathrm{s}$ in two clusters in the left hemisphere (only in the antipsychotic-treated subgroup). When contrasting the medicated SP's with HCs, three much larger clusters became apparent, again, mainly in the left hemisphere, suggesting that antipsychotic exposure may largely account for the increments in $\mathrm{t}-\mathrm{Cr}$. Additionally in medicated SP's, $\mathrm{t}$-Cho was increased in a left frontal region. Finally, NAA and MINO were reduced only in antipsychotic-naïve SP's vs HC's. Given these novel findings, this study demonstrates the feasibility and utility of 3-dimensional EPSI to explore schizophrenia vs antipsychoticrelated neurometabolic findings, in a manner that would not be possible with single-voxel ${ }^{1} \mathrm{H}-\mathrm{MRS}$.

Only two ${ }^{1} \mathrm{H}$-MRS studies have investigated the STG in schizophrenia. One examined chronically-treated schizophrenia and bipolar-I (BP-I) patients with single voxels over STG bilaterally at 3T [21]. They found reduced glutamate in the left STG in BP-I but only marginal reductions in schizophrenia $(p=$ 0.1 ). The other study used 2-dimensional ${ }^{1} \mathrm{H}$-MRSI at $1.5 \mathrm{~T}$ and failed to detect Glx differences in STG [22]. The STG has been implicated in the development of psychosis in structural MRI studies. Longitudinal STG volume reductions were reported in ultra high-risk subjects who converted to psychosis, but not in non-converters [23]. Furthermore, first episode psychosis subjects also had progressive STG reductions which were more prominent in the left side and correlated with severity of delusions [23]. Functional and pharmacological studies have further implicated the STG in schizophrenia. Acute NMDAR hypofunction, a model for schizophrenia, reduces mismatch negativity (MMN), an evoked response which occurs in the 


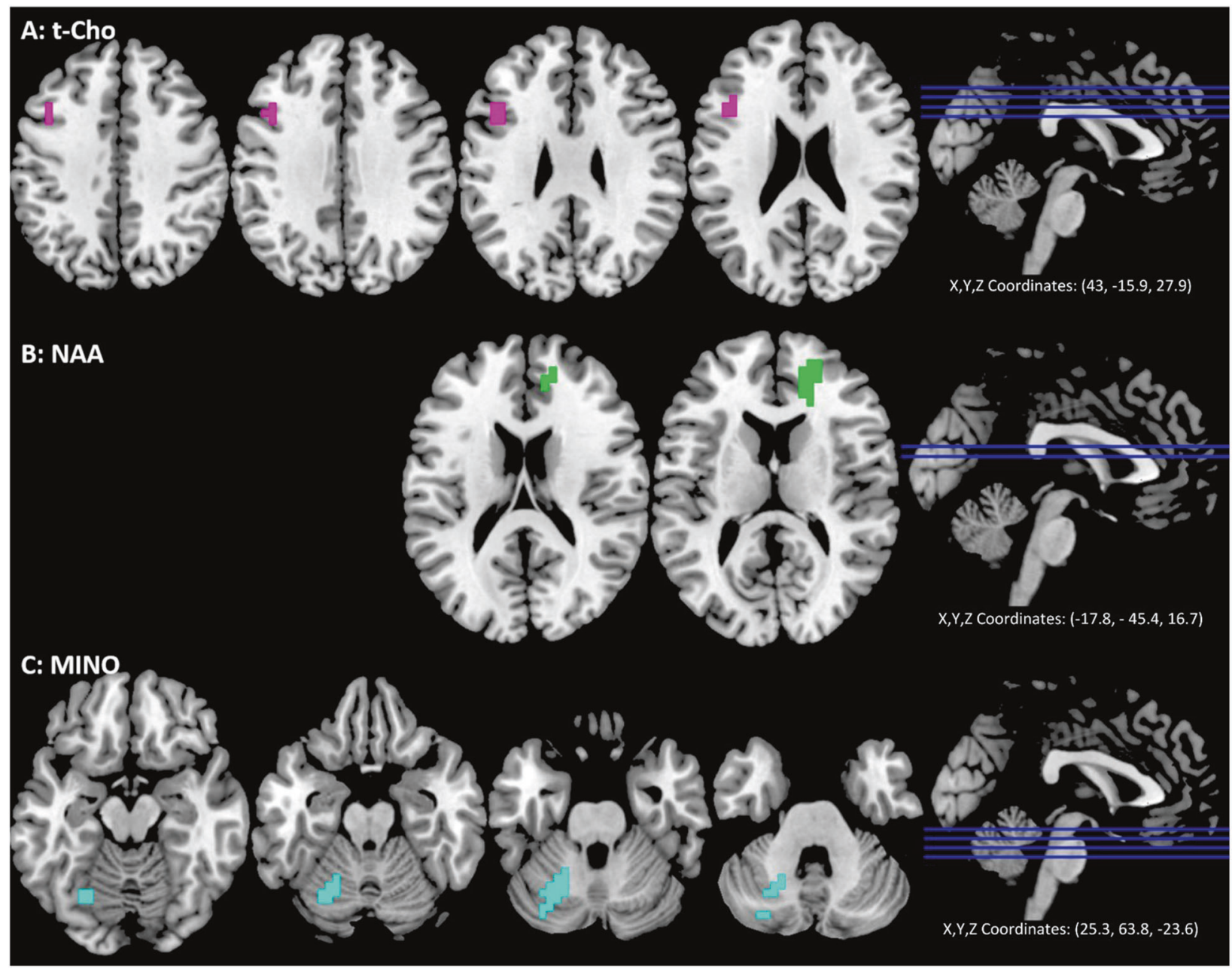

Fig. 4 Abnormalities in total choline (t-Cho), $\mathbf{N}$-acetylaspartate (NAA) and myo-inositol (MINO) in schizophrenia. Row a Increased t-Cho (shown in pink) in antipsychotic-treated SP vs HC in one cluster $(18$ voxels, CCLAV $=0.04)$ in mainly the left middle frontal gyrus. Row b Reduced NAA (shown in green) in antipsychotic-naive SP vs HC in one cluster (19 voxels, CCLAV $=0.05$ ) in mainly right medial, superior frontal gyri and middle frontal white matter. Row c Reduced myo-inositol (shown in light blue) in antipsychotic-naive SP vs HC in one cluster (34 voxels, CCLAV $=0.02$ ) in mainly the left cerebellum.

process of comparing sound deviations, and that originates from STG [24]. MMN is impaired in schizophrenia, as well as in highrisk subjects who convert to psychosis [25]. Also, acute NMDAR hypofunction with ketamine induces schizophrenia-like symptoms and cognitive deficits in healthy volunteers [25]. Acute ketamine also increases glutamate in medial frontal cortex [26]. However, a 1 month exposure of low dose $(2.58 \mathrm{mg} / \mathrm{kg} /$ day $)$ phencyclidine resulted in medio-frontal glutamate reductions in rats [27]. Schizophrenia, even amongst most "first episode" patients, is a chronic illness, so acute ketamine exposure may not adequately model the persistent developmental effects of NMDAR hypofunction. In postmortem studies, increments in calcineurin, a protein activated by $\mathrm{Ca}^{++}$in-flow through NMDAR, were reported in STG across layers II-VI [28]. Also increased protein insolubility was found in STG and frontal cortex in schizophrenia [29]. Recently, reductions in synaptosome levels of post-synaptic excitatory proteins were reported in primary auditory cortex of schizophrenia [30], consistent with synaptic glutamatergic hypofunction. Hence, this literature converges with our findings to support that left STG abnormalities with early hypoglutamatergic dysfunction may be a trait characteristic of schizophrenia. The reduced Glx may be secondary to dendritic retraction (seen in post-mortem studies [31]), caused by a "cold" excitotoxic process due to bouts of increased synaptic glutamate. Alternatively, reduced Glx in left
STG may represent the effects of a primary NMDAR hypofunction in a region critical for sound discrimination and language.

Though some single-voxel studies have reported increased $\mathrm{t}-\mathrm{Cr}$ $[32,33]$, these have not been replicated [34]. However, an ${ }^{1} \mathrm{H}-\mathrm{MRSI}$ study of the hippocampus revealed increased $\mathrm{t}-\mathrm{Cr}$ in chronicallymedicated schizophrenia [35]. We found increased t-Cr only in antipsychotic-treated patients. Antipsychotics are known to cause acute changes increasing subcortical and decreasing cortical metabolism [36, 37]. Furthermore, a reduction in the forward rate constant of creatine kinase, the enzyme that converts $\mathrm{Cr}$ to $\mathrm{P}-\mathrm{Cr}$, was reported in medicated schizophrenia [38]. Hence, it is possible that the increase in $\mathrm{t}-\mathrm{Cr}$ represents an adaptation to the lowering cortical metabolic effects of antipsychotics, by increasing the total pool of creatine available for energetic transfer. Regarding t-Cho, alterations have not been consistently reported in single-voxel studies [34]. We speculate that increased t-Cho only in treated patients suggests an adaptive glial response to antipsychotics in the left medial frontal cortex. Investigations on inositol in schizophrenia have been sparse but a recent meta-analysis reported reductions in medial frontal regions in predominantly treated patients [39]. However, cerebellar or other regions were not examined. Our results suggest reduced cerebellar glial function in schizophrenia, but will require replication.

Reduced NAA has been repeatedly found in schizophrenia [34]. In the dorsal AC in particular, both NAA and glutamate were 
reduced in the largest study of medicated first episode psychosis patients at 7T [40]. These results were replicated in a smaller sample of treated early schizophrenia subjects also at $7 \mathrm{~T}$ [41]. Our findings of dAC NAA reduction only in the antipsychotic-naïve subgroup suggests a normalizing effect of treatment (only $3 / 19$ voxels in the reduced cluster, were significantly lower $(p<0.001)$ in antipsychotic-treated SPs vs HCs). It is also possible that the better signal-to-noise at 7T allows the detection of persistent NAA reductions in the $\mathrm{dAC}$ in treated patients $[40,41]$.

Although a few previous studies have used whole-brain ${ }^{1} \mathrm{H}$ MRSI in schizophrenia, we are not aware of any using a TE short enough to examine Glx. One reported lower NAA in broad white matter regions in chronically ill subjects [42]. Another found reduced $\mathrm{NAA} / \mathrm{t}-\mathrm{Cr}$ but examined only selected voxels overlapping with the hippocampus and the dorsolateral prefrontal cortex [43]. Neither of the previous whole-brain ${ }^{1} \mathrm{H}$-MRSI studies systematically examined all voxels acquired. To do so, normalization of all voxels in a common space and accounting for multiple comparisons are necessary, which are standard in other neuroimaging modalities (eg: MRI, DTI, fMRI). In the present study we demonstrate that these methods can be applied to 3-dimensional ${ }^{1} \mathrm{H}$-MRS.

Our findings did not support the hypothesized Glx increase in $\mathrm{dAC}$ and in the dorsal striatum in schizophrenia. Unfortunately there were not enough voxels with good quality spectra encompassing the dorsal striatum to measure Glx (see mask Fig. 1). For the $d A C$, we did have adequate coverage. In this region two recent and much larger studies reported opposite changes in medicated samples: increased Glx in schizophrenia (SP's, N=104 [6]) and reduced glutamate in first episode psychosis (Psychoses, $N=81$ [40]). The present study, which included young individuals with differences in antipsychotic exposure, may have been underpowered to detect $\mathrm{dAC}$ group differences.

This study has several strengths. Volumetric EPSI allowed examination of most of the brain including numerous gray and white matter regions known to be involved in schizophrenia [44]. Voxels were automatically selected based on standard spectral quality criteria. We chose AFNI for analyses because it allows introduction of co-variates at the voxel level, a critical issue in ${ }^{1} \mathrm{H}-\mathrm{MRS}$ analyses since the gray/white matter tissue effect is much larger than age or diagnostic group effects [6]. The sample studied included similar numbers of antipsychotic-naïve and treated young schizophrenia subjects. However, limitations must be acknowledged. First, the spectral resolution obtained at $3 \mathrm{~T}$ did not allow reliable discrimination of glutamine from glutamate. Second, though whole-brain EPSI was acquired, brain areas with greater magnetic field inhomogeneity (eg: orbitofrontal regions, frontal and temporal poles and base of the brain, including dorsal striatum) did not yield consistent spectral quality and so could not be included in analyses. These are regions potentially important for schizophrenia. Although more severe, these challenges are not exclusive to three-dimensional ${ }^{1} \mathrm{H}-\mathrm{MRSI}$. For example, other more standard neuro-imaging modalities in the Connectome Project, also found suboptimal brain coverage in some of these regions [45]. Clearly, single-voxel ${ }^{1} \mathrm{H}-\mathrm{MRS}$ allows a scanning protocol to be tailored to a specific brain region, making data from these difficult regions more accessible. However, in schizophrenia structural studies detect involvement of a majority of the brain early in the illness [2]. Therefor spatial coverage in the several thousands of voxels offers some advantages over the usual single-voxel approaches. Third, our samples of 19 medication-naïve and 17 treated SP are relatively small, and this is particularly relevant to the likelihood of type-II error in the examination of the dAC. Medication status can clearly have an effect [46]. Fourth, we did not statistically correct for examining five metabolites. Finally, this was a cross-sectional study, so mechanistic interpretations are inherently limited.

In summary, a data-driven examination with three-dimensional EPSI in early schizophrenia revealed a reduction in glutamate metabolism (Glx) in the left STG/MTG regardless of antipsychotic status and a larger mostly left-sided area of increased t-Cr, only in medicated patients. Replication of these findings would support mechanistic postmortem studies and therapeutic neuromodulation studies of the left STG/MTG in schizophrenia. Also, the feasibility of our three-dimensional brain ${ }^{1} \mathrm{H}$-MRSI analysis approach is demonstrated in a psychiatric population. Finally, caution is warranted when referencing ${ }^{1} \mathrm{H}$-MRS metabolite values to $\mathrm{t}-\mathrm{Cr}$ in studies of treated schizophrenia populations.

\section{FUNDING AND DISCLOSURE}

$J R B$ received royalties from Up-to-date. JU reported no biomedical financial interests or potential conflicts of interest. GM reported no biomedical financial interests or potential conflicts of interest. TJ reported no biomedical financial interests or potential conflicts of interest. AAM reported no biomedical financial interests or potential conflicts of interest. MT was an employee of Lilly (1997-2008) and has received honoraria from or consulted for Abbott, AstraZeneca, Alkermes, Allergan, Bristol Myers Squibb, GlaxoSmithKline, Lilly, Johnson \& Johnson, Otsuka, Merck, Gedeon Richter Plc, Sunovion, Forest, Roche, Elan, Lundbeck, Teva, Pamlab, Minerva, Neurocrine, Pfizer, Wyeth and Wiley Publishing; his spouse was a full time employee at Lilly (1998-2013). CG reported no biomedical financial interests or potential conflicts of interest. RL reported no biomedical financial interests or potential conflicts of interest. Supported by NIMH R01MH084898 to JRB and 1 P20 RR021938-01A1 and DHHS/ $\mathrm{NIH/NCRR} 3$ UL1 RR031977-02S2. The MIDAS software was supported by R01 EB016064. The authors declare no competing interests.

\section{ACKNOWLEDGEMENTS}

We are grateful to Nattida Payaknait and Nicholas Lemke, employees of the UNM Department of Psychiatry and to Diana South and Cathy Smith, MRN employees, for their contributions with data collection.

\section{AUTHOR CONTRIBUTIONS}

JRB was involved in the design, acquisition, analysis, interpretation of data for the work, drafting, and revising of the paper. JU was involved in the analysis, interpretation of data for the work, drafting and revising of the paper. GM was involved in the analysis, interpretation of data for the work, drafting, and revising of the manuscript. TJ was involved in the analysis, interpretation of data for the work, drafting, and revising of the paper. AAM was involved in the interpretation of data for the work, drafting and revising of the paper. MT was involved in the interpretation of data for the work, drafting and revising of the paper. CG was involved in the interpretation of data for the work, drafting, and revising of the paper. RL was involved in the design, acquisition, analysis, interpretation of data for the work, drafting and revising of the paper.

\section{ADDITIONAL INFORMATION}

Supplementary Information accompanies this paper at (https://doi.org/10.1038/ s41386-020-0707-y).

Publisher's note Springer Nature remains neutral with regard to jurisdictional claims in published maps and institutional affiliations.

\section{REFERENCES}

1. Moghaddam B, Javitt D. From revolution to evolution: the glutamate hypothesis of schizophrenia and its implication for treatment. Neuropsychopharmacology. 2012;37:4-15.

2. Olabi B, Ellison-Wright I, McIntosh AM, Wood SJ, Bullmore E, Lawrie SM. Are there progressive brain changes in schizophrenia? A meta-analysis of structural magnetic resonance imaging studies. Biol Psychiatry. 2011;70:88-96.

3. Marsman A, van den Heuvel MP, Klomp DW, Kahn RS, Luijten PR, Hulshoff Pol HE. Glutamate in schizophrenia: a focused review and meta-analysis of (1)H-MRS studies. Schizophr Bull. 2013;39:120-9.

4. Merritt K, Egerton A, Kempton MJ, Taylor MJ, McGuire PK. Nature of glutamate alterations in schizophrenia: a meta-analysis of proton magnetic resonance spectroscopy Studies. JAMA Psychiatry. 2016;73:665-74. 
5. Iwata Y, Nakajima S, Plitman E, Mihashi Y, Caravaggio F, Chung JK, et al. Neurometabolite levels in antipsychotic-naive/free patients with schizophrenia: a systematic review and meta-analysis of (1)H-MRS studies. Prog Neuropsychopharmacol Biol Psychiatry. 2018;86:340-52.

6. Bustillo JR, Jones T, Chen H, Lemke N, Abbott C, Qualls C, et al. Glutamatergic and neuronal dysfunction in gray and white matter: a spectroscopic imaging study in a large schizophrenia sample. Schizophr Bull. 2016;43:611-19.

7. de la Fuente-Sandoval C, Leon-Ortiz P, Azcarraga $M$, Stephano S, Favila $R$ Diaz-Galvis $L$, et al. Glutamate levels in the associative striatum before and after 4 weeks of antipsychotic treatment in first-episode psychosis: a longitudinal proton magnetic resonance spectroscopy study. JAMA Psychiatry. 2013;70:1057-66.

8. Buchanan RW, Davis M, Goff D, Green MF, Keefe RS, Leon AC, et al. A summary of the FDA-NIMH-MATRICS workshop on clinical trial design for neurocognitive drugs for schizophrenia. Schizophr Bull. 2005;31:5-19.

9. Kay SR, Fiszbein A, Opler LA. The positive and negative syndrome scale (PANSS) for schizophrenia. Schizophr Bull. 1987;13:261-76.

10. Goryawala MZ, Sheriff $S$, Maudsley AA. Regional distributions of brain glutamate and glutamine in normal subjects. NMR Biomed. 2016;29:1108-16.

11. Maudsley AA, Domenig C, Govind V, Darkazanli A, Studholme C, Arheart K, et al. Mapping of brain metabolite distributions by volumetric proton MR spectroscopic imaging (MRSI). Magn Reson Med. 2009;61:548-59.

12. Cox RW. AFNI: software for analysis and visualization of functional magnetic resonance neuroimages. Comput Biomed Res. 1996;29:162-73.

13. Vaden KI Jr., Gebregziabher M, Kuchinsky SE, Eckert MA. Multiple imputation of missing $\mathrm{fMRI}$ data in whole brain analysis. Neuroimage. 2012;60:1843-55.

14. Maudsley AA, Darkazanli A, Alger JR, Hall LO, Schuff N, Studholme C, et al. Comprehensive processing, display and analysis for in vivo MR spectroscopic imaging. NMR Biomed. 2006;19:492-503.

15. Ebel A, Maudsley AA. Improved spectral quality for 3D MR spectroscopic imaging using a high spatial resolution acquisition strategy. Magn Reson imaging. 2003:21:113-20.

16. Goryawala M SM, Maudsley AA. Effects of Apodization smoothing and denoising on spectral fitting. Magn Reson Med. 2020;20:108-14.

17. Soher BJ, Young K, Govindaraju V, Maudsley AA. Automated spectral analysis III: application to in vivo proton MR spectroscopy and spectroscopic imaging. Magn Reson Med. 1998;40:822-31.

18. Zhang Y, Taub E, Salibi N, Uswatte G, Maudsley AA, Sheriff S, et al. Comparison of reproducibility of single voxel spectroscopy and whole-brain magnetic resonance spectroscopy imaging at 3T. NMR Biomed. 2018;31:e3898.

19. Rubin LH, Witkiewitz K, Andre JS, Reilly S. Methods for handling missing data in the behavioral neurosciences: don't throw the baby rat out with the bath water. J Undergrad Neurosci Educ. 2007;5:A71-7.

20. Cox RW, Chen G, Glen DR, Reynolds RC, Taylor PA. FMRI clustering in AFNI: falsepositive rates redux. Brain Connect. 2017;7:152-71.

21. Atagun MI, Sikoglu EM, Can SS, Karakas-Ugurlu G, Ulusoy-Kaymak S, Caykoylu A, et al. Investigation of Heschl's gyrus and planum temporale in patients with schizophrenia and bipolar disorder: a proton magnetic resonance spectroscopy study. Schizophr Res. 2015;161:202-9.

22. Seese RR, O'Neill J, Hudkins M, Siddarth P, Levitt J, Tseng B, et al. Proton magnetic resonance spectroscopy and thought disorder in childhood schizophrenia. Schizophr Res. 2011;133:82-90.

23. Takahashi T, Wood SJ, Yung AR, Soulsby B, McGorry PD, Suzuki M, et al. Progressive gray matter reduction of the superior temporal gyrus during transition to psychosis. Arch Gen Psychiatry. 2009;66:366-76.

24. Javitt DC, Steinschneider M, Schroeder CE, Arezzo JC. Role of cortical N-methyl-Daspartate receptors in auditory sensory memory and mismatch negativity generation: implications for schizophrenia. Proc Natl Acad Sci USA. 1996;93:11962-7.

25. Javitt DC, Sweet RA. Auditory dysfunction in schizophrenia: integrating clinical and basic features. Nat Rev Neurosci. 2015;16:535-50.

26. Stone JM, Dietrich C, Edden R, Mehta MA, De Simoni S, Reed LJ, et al. Ketamine effects on brain GABA and glutamate levels with 1H-MRS: relationship to ketamine-induced psychopathology. Mol Psychiatry. 2012;17:664-5.
27. Bustillo J, Galloway MP, Ghoddoussi F, Bolognani F, Perrone-Bizzozero N. Medialfrontal cortex hypometabolism in chronic phencyclidine exposed rats assessed by high resolution magic angle spin $11.7 \mathrm{~T}$ proton magnetic resonance spectroscopy. Neurochemistry Int. 2012;61:128-31.

28. Wada A, Kunii Y, Matsumoto J, Hino M, Yang Q, Niwa Sl, et al. Prominent increased calcineurin immunoreactivity in the superior temporal gyrus in schizophrenia: A postmortem study. Psychiatry Res. 2017;247:79-83.

29. Nucifora LG, MacDonald ML, Lee BJ, Peters ME, Norris AL, Orsburn BC, et al. Increased protein insolubility in brains from a subset of patients with schizophrenia. Am J Psychiatry. 2019;176:730-43.

30. MacDonald ML, Garver M, Newman J, Sun Z, Kannarkat J, Salisbury R, et al Synaptic proteome alterations in the primary auditory cortex of individuals with schizophrenia. JAMA Psychiatry. 2019:77;1-10.

31. Glantz LA, Lewis DA. Decreased dendritic spine density on prefrontal cortical pyramidal neurons in schizophrenia. Arch Gen Psychiatry. 2000;57:65-73.

32. Ongur D, Prescot AP, Jensen JE, Cohen BM, Renshaw PF. Creatine abnormalities in schizophrenia and bipolar disorder. Psychiatry Res. 2009;172:44-8.

33. Tibbo PG, Bernier D, Hanstock CC, Seres P, Lakusta B, Purdon SE. 3-T proton magnetic spectroscopy in unmedicated first episode psychosis: a focus on creatine. Magn Reson Med. 2013;69:613-20.

34. Kraguljac NV, Reid M, White $D$, Jones R, den Hollander J, Lowman $D$, et al. Neurometabolites in schizophrenia and bipolar disorder - a systematic review and meta-analysis. Psychiatry Res. 2012;203:111-25.

35. Meyer EJ, Kirov II, Tal A, Davitz MS, Babb JS, Lazar M, et al. Metabolic abnormalities in the hippocampus of patients with schizophrenia: A 3D multivoxel MR spectroscopic imaging study at 3T. AJNR Am J Neuroradiol. 2016;37:2273-9.

36. Lahti AC, Holcomb HH, Weiler MA, Medoff DR, Tamminga CA. Functional effects of antipsychotic drugs: comparing clozapine with haloperidol. Biol Psychiatry. 2003;53:601-8.

37. Lahti AC, Weiler MA, Medoff DR, Tamminga CA, Holcomb HH. Functional effects of single dose first- and second-generation antipsychotic administration in subjects with schizophrenia. Psychiatry Res 2005;139:19-30.

38. Du F, Cooper AJ, Thida T, Sehovic S, Lukas SE, Cohen BM, et al. In vivo evidence for cerebral bioenergetic abnormalities in schizophrenia measured using 31P magnetization transfer spectroscopy. JAMA Psychiatry. 2014;71:19-27.

39. Das TK, Dey A, Sabesan P, Javadzadeh A, Theberge J, Radua J, et al. Putative astroglial dysfunction in schizophrenia: a meta-analysis of (1)H-MRS studies of medial prefrontal myo-inositol. Front Psychiatry. 2018;9:438.

40. Wang AM, Pradhan S, Coughlin JM, Trivedi A, DuBois SL, Crawford JL, et al. Assessing brain metabolism with 7-T proton magnetic resonance spectroscopy in patients with first-episode psychosis. JAMA Psychiatry. 2019;76:314-23.

41. Reid MA, Salibi N, White DM, Gawne TJ, Denney TS, Lahti AC. 7T Proton magnetic resonance spectroscopy of the anterior cingulate cortex in first-episode schizophrenia. Schizophr Bull. 2019;45:180-9.

42. Lim KO, Adalsteinsson E, Spielman D, Sullivan EV, Rosenbloom MJ, Pfefferbaum A. Proton magnetic resonance spectroscopic imaging of cortical gray and white matter in schizophrenia. Arch Gen Psychiatry. 1998;55:346-52.

43. Bertolino A, Nawroz S, Mattay VS, Barnett AS, Duyn JH, Moonen CT, et al. Regionally specific pattern of neurochemical pathology in schizophrenia as assessed by multislice proton magnetic resonance spectroscopic imaging. Am J Psychiatry. 1996;153:1554-63.

44. Haijma SV, Van Haren N, Cahn W, Koolschijn PC, Hulshoff Pol HE, Kahn RS. Brain volumes in schizophrenia: a meta-analysis in over 18000 subjects. Schizophr Bull. 2013;39:1129-38.

45. Glasser MF, Coalson TS, Robinson EC, Hacker CD, Harwell J, Yacoub E, et al. A multi-modal parcellation of human cerebral cortex. Nature. 2016;536:171-8.

46. Kegeles LS, Mao X, Stanford AD, Girgis R, Ojeil N, Xu X, et al. Elevated prefrontal cortex gamma-aminobutyric acid and glutamate-glutamine levels in schizophrenia measured in vivo with proton magnetic resonance spectroscopy. Arch Gen Psychiatry. 2012;69:449-59.

47. Gardner DM, Murphy AL, O'Donnell H, Centorrino F, Baldessarini RJ. International consensus study of antipsychotic dosing. Am J Psychiatry. 2010;167:686-93. 